\title{
Performance comparison of three models for thermal property determination from experimental phase change data
}

\author{
Heinrich Badenhorst* \\ SARChI Chair in Carbon Materials and Technology, Department of Chemical Engineering, University \\ of Pretoria, Lynnwood Road, Pretoria, 0002, South Africa
}

\begin{abstract}
The characterization of thermal properties continues to be a challenge facing the development of composite phase change materials. To overcome the practical issues of current techniques a new experimental method was developed. The system is modelled using two fundamental models and a simplified analytical representation. Pure myristic acid is used for validation and a performance comparison based on time, accuracy and stability. All three approaches perform comparatively well in terms of accuracy with an average prediction error of less than one third of a degree and all thermal properties in line with expectations. The enthalpy model is susceptible to instability while the effective heat capacity method does not show any such behaviour. In addition, due to the choice of a smooth function for the transition from liquid to solid, this method delivers more realistic behaviour. The effective heat capacity method is faster than the enthalpy method by a factor of three, but the analytical approach is the fastest. However for a more robust and comprehensive representation the effective heat capacity method is preferred.
\end{abstract}

Keywords: effective heat capacity, enthalpy, thermal properties

\section{Introduction}

Globally there is a growing focus on developing renewable energy options [1, 2]. One of the most significant difficulties facing wide scale implementation of renewable energy is reliable storage [3]. Due to the inconsistent and/or cyclic nature

\footnotetext{
* Corresponding author. Tel: +27 12420 4989. Fax: +27 12420 2516. E-mail: carbon@up.ac.za
} 
of renewable resources, collection and storage is essential. In general the availability of renewable energy does not coincide with the periods of peak energy consumption. To ensure that the burden on non-renewable reserves are reduced efficient storage and prompt availability is the key to increasing the utilization of renewable options.

Some of the promising possibilities for the storage of solar thermal energy are phase change materials (PCMs) [4]. Due to the utilization of latent heat rather than sensible these materials have a very high energy density. During the transition from solid to liquid energy is stored and then recovered during the reverse transformation. However, the low thermal conductivity of these materials is a significant deficiency [5]. To overcome this limitation recent research has increasingly focused on developing composites, to improve the thermal performance of the PCMs [6-11].

For this process to be effective, the rapid and accurate characterization of these materials is crucial. Not only for optimizing parameters such as additive loading [12] but also predicting material behaviour in real systems [13]. Characterization techniques for individual properties do exist, such as Differential Scanning Calorimetry (DSC) for enthalpy/heat capacity and the transient hot wire (THW) method for thermal conductivity. However they are susceptible to some practical issues such as sample size and heterogeneity. Most commercial DSC devices use milligram size samples which are not always representative of bulk behaviour and some heat transfer phenomena may be ignored [14]. Most of the composite materials and even the PCMs themselves are porous solids, in many cases comprised of particulates or matrices. For the THW technique to deliver a representative measurement the sample should be homogenous [15], which is usually not the case. Furthermore sample penetration is very limited which may result in incorrect results for composites $[16,17]$ since they can contain some surface inhomogeneity due to processing.

The aim of this research is to develop a platform for the rapid assessment of PCMs and composites. To this end a straightforward prototype system was developed for future use as a routine method to characterize PCM composites. The setup was developed specifically to overcome the aforementioned practical issues. The design is novel but similar to the widely used $t$-method [18]. To ensure that the parametric estimates are valid, a comprehensive, fundamental model must be developed which 
accounts for all the relevant phenomena. Only in this manner can assumptions be validated and the system behaviour be fully understood.

The systems under consideration invariably contain mixtures of at least two phases. This makes them problematic to represent mathematically and hence simulate in a rapid and simple manner. Over the years this has led to a proliferation of models for numerous geometries (Cartesian, cylindrical, spherical) and configurations (packed bed, finned surfaces, porous materials, etc.). Many reviews on the subject have been published [14, 19-26]. Unfortunately however, as pointed out by Dutil et al. [26] recent studies are rarely validated using experimental results. In addition, since they are only benchmarked against other numerical studies, the time taken to fit experimental data is not considered. This is not the same as the time required for a single simulation run. Many simulation runs may be required to optimally fit the model to experimental data depending on the manner and speed in which the fitting procedure converges.

Broadly speaking the current modelling approaches can be classified into two groups: enthalpy based methods and the effective heat capacity methods [25]. From a thermodynamic perspective both approaches are very similar. The primary difference is the definition of a melt fraction for the enthalpy method, whereas the effective heat capacity method uses a temperature based heat capacity function. Both approaches have inherent disadvantages such as difficulties in handling discontinuous thermal properties for the enthalpy [27] and cases with a narrow phase transition zone for the effective heat capacity [25] method respectively. Very few studies have focused on comparing the performance of these two schemes and less, if any, on their relative ability to fit real experimental data. Another factor which is rarely considered is the stability of these methods in the two phase region.

The objective of this investigation is to validate the new measuring device and compare the performance of the enthalpy method and a slightly modified effective heat capacity approach. A finite difference model was used to fit the experimental data for a typical PCM obtained using the prototype system. As the first step in validation, pure myristic acid was chosen as a reference material since it has been extensively studied before [12, 28-33]. The main criteria for assessment are convergence time, accuracy and stability. The parametric values are compared to 
expected values from literature as well as DSC and THW measurements. The influence of certain adjustable parameters on the fit are also analysed.

Due to the low thermal conductivity of the sample, experimental tests can take several hours, routinely longer than eight. The secondary objective is to use the insights gained from the data and fundamental models to propose a suitable simplified system representation. It is then evaluated whether this approach can substantially reduce test and model convergence time whilst still maintaining an acceptable level of accuracy. If satisfactory this technique may provide a very simple and rapid system for assessing PCMs and composites.

\section{Theoretical modelling}

The classical representation of the enthalpy-temperature relationship and its derivative are given in Figure 1.

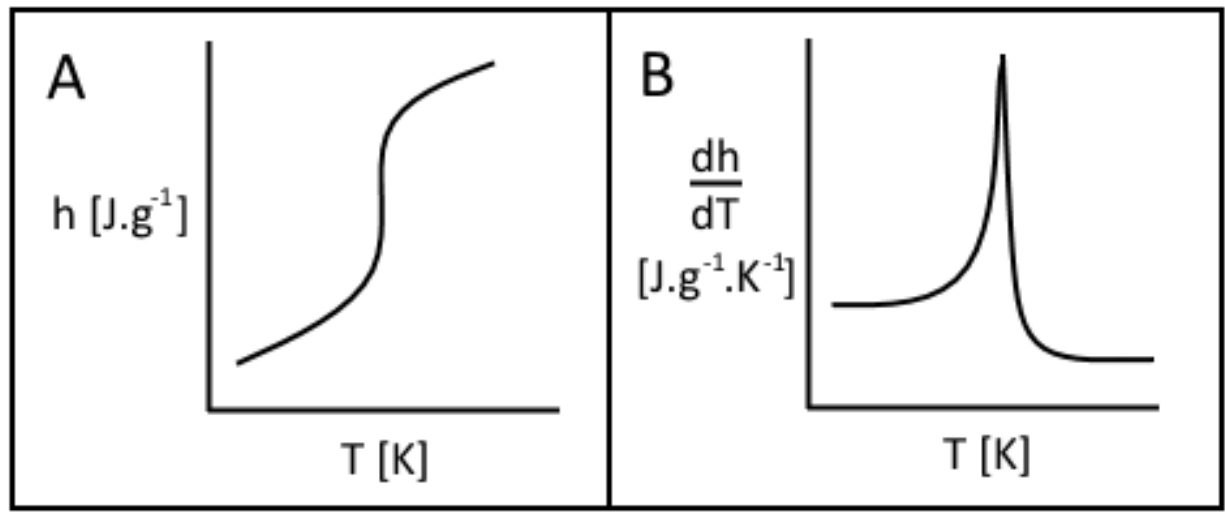

Figure 1: Enthalpy as a function of temperature

From a thermodynamic point of view, the peak in Figure 1 (B) has infinite height and zero width. Its integral gives the value of the phase change enthalpy, whilst the horizontal end-points represent the solid and liquid phase heat capacities. From a practical perspective the more gradual representation in Figure 1 is closer to the true behaviour since real transitions have a kinetic aspect. For example due to nucleation and growth phenomena these transformations do not occur instantaneously. Since the integral of the normal distribution is one, the peak region of Figure 1 (B) could be represented by the normal distribution multiplied by the phase change enthalpy. Furthermore if the entire curve is divided by the solid heat capacity value, the left 
intercept would be one. This dimensionless function, $g(\mathrm{~T})$, the derivative of the enthalpy divided by the solid heat capacity, is shown in Figure 2:

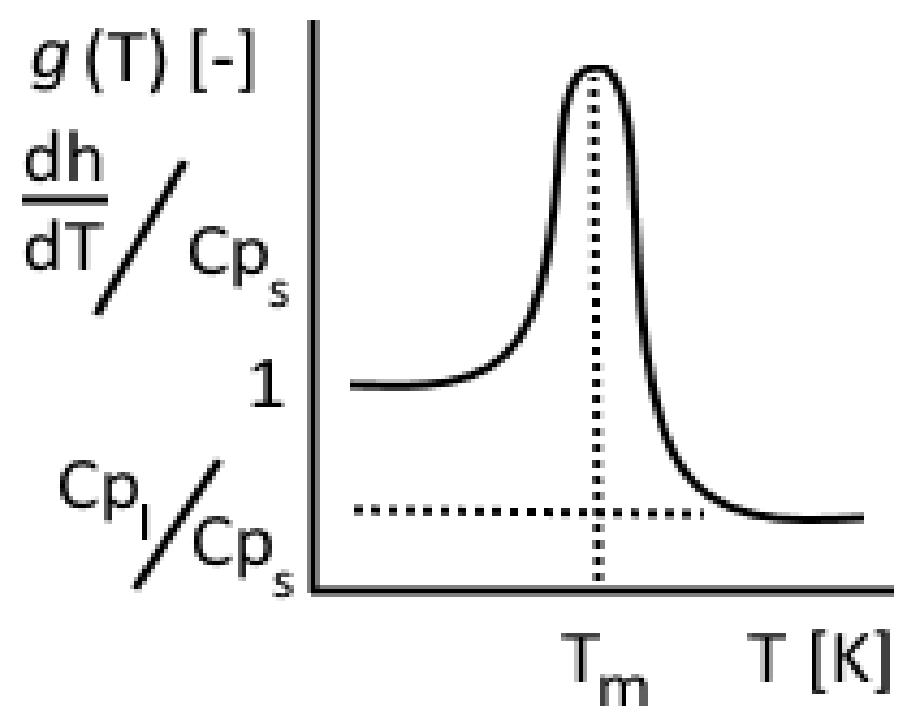

Figure 2: Dimensionless enthalpy derivative as a function of temperature

This approximation is very similar to the conventional effective heat capacity method. In this case a continuous function employing the normal distribution was substituted for the traditional discontinuous heat capacity function given by [25]:

$$
C_{e f f}=\left\{\begin{array}{cc}
C p_{s} & T<T_{1} \\
\frac{\Delta H}{T_{2}-T_{1}} & T_{1} \leq T \leq T_{2} \\
C p_{l} & T>T_{2}
\end{array}\right.
$$

This approach makes implementation slightly easier and more representative since there are no sudden transitions or discontinuities. The changeover from solid to liquid heat capacity has a relatively minor effect since the change in their ratio is small compared to the peak height. This is determined by the phase change enthalpy $(\Delta H)$ which is overwhelming as long as the normal distribution is not excessively wide around the melting temperature. Based on DSC results a realistic transition zone of one degree was chosen. The integral of the same normal distribution function can be used to represent the change in other properties, for example density and thermal conductivity during phase change, as demonstrated in Figure 3. 


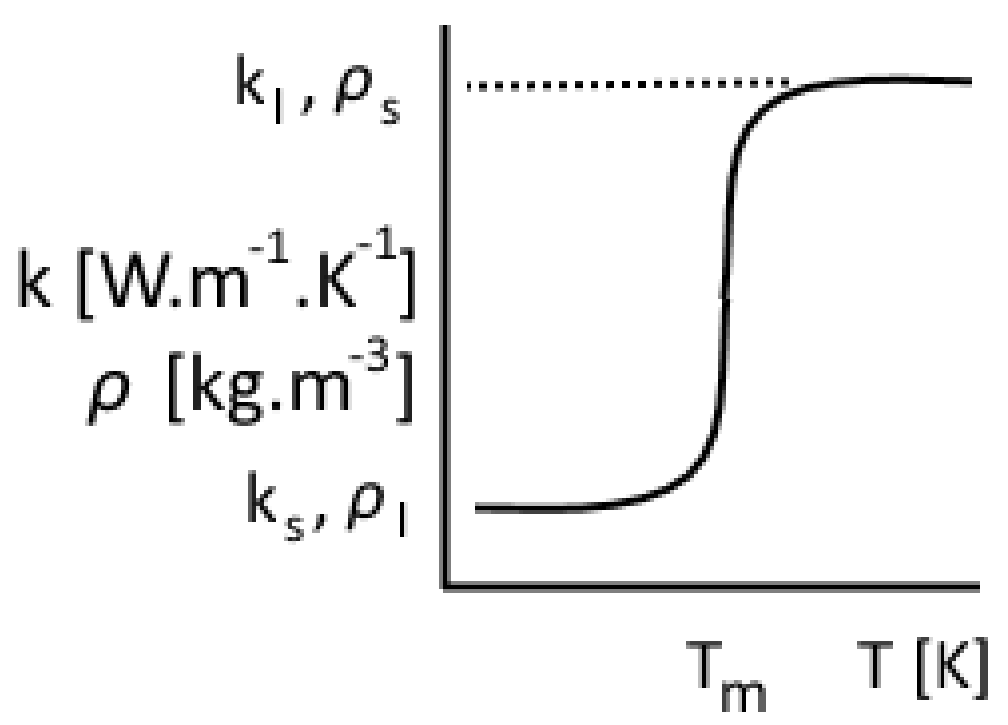

Figure 3: Density and thermal conductivity as a function of temperature

To obtain the finite difference equations the energy balance may be applied to a one dimensional segment which gives:

$$
\nabla k \nabla T=\frac{\partial}{\partial x} k \frac{\partial T}{\partial x}=\rho \frac{\partial h}{\partial t}=\rho \frac{\partial h}{\partial T} \frac{\partial T}{\partial t}
$$

This is equivalent to:

$$
\frac{\partial}{\partial x} k \frac{\partial T}{\partial x}=\rho C p_{s} g(T) \frac{\partial T}{\partial t}
$$

Since $g(T)$ is a now a known continuous function, this expression can simply be discretized and applied to the given geometry. A full derivation including the boundary conditions is given in Appendix A. This represents the effective heat capacity method, for the enthalpy method the time differential of the enthalpy is expanded in a slightly different manner [34]. In this case the total enthalpy $(H)$ is specified as the sum of the sensible and latent heats, i.e.

$$
H(T)=\int_{T}^{T_{m}} \rho_{s} C p_{s} d T+\int_{T_{m}}^{T} \rho_{l} C p_{l} d T+f(T) \Delta H
$$

Phase change is assumed to occur isothermally, where the liquid melt fraction is given by:

$$
f(T)= \begin{cases}0 & T<T_{m} \\ 0 \sim 1 & T=T_{m} \\ 1 & T>T_{m}\end{cases}
$$

Thus the energy balance becomes:

$$
\nabla k \nabla T=\frac{\partial}{\partial x} k \frac{\partial T}{\partial x}=\rho \frac{\partial h}{\partial t}=\rho C p \frac{\partial T}{\partial t}+\Delta H \frac{\partial f(T)}{\partial t}
$$


The solution of this expression is slightly more complicated as the temperature is kept constant once the melting point is reached. Then the melt fraction must be solved for iteratively whilst satisfying the appropriate implicit difference equation, derived in Appendix A. One possibility for representing the property transition from solid to liquid (heat capacity, density and thermal conductivity) is through the use of the melt fraction itself, for example:

$$
C p=C p_{l} f(T)+C p_{s}[1-f(T)]
$$

This is slightly different from the original scheme proposed by Voller [27], whereby the properties instantaneously transition from liquid to solid for a given node when the melt fraction decreases below 0.5. Initially a two dimensional model was used, but subsequently a spherical model was found to be sufficient. The reason can be ascertained from Figure 4.

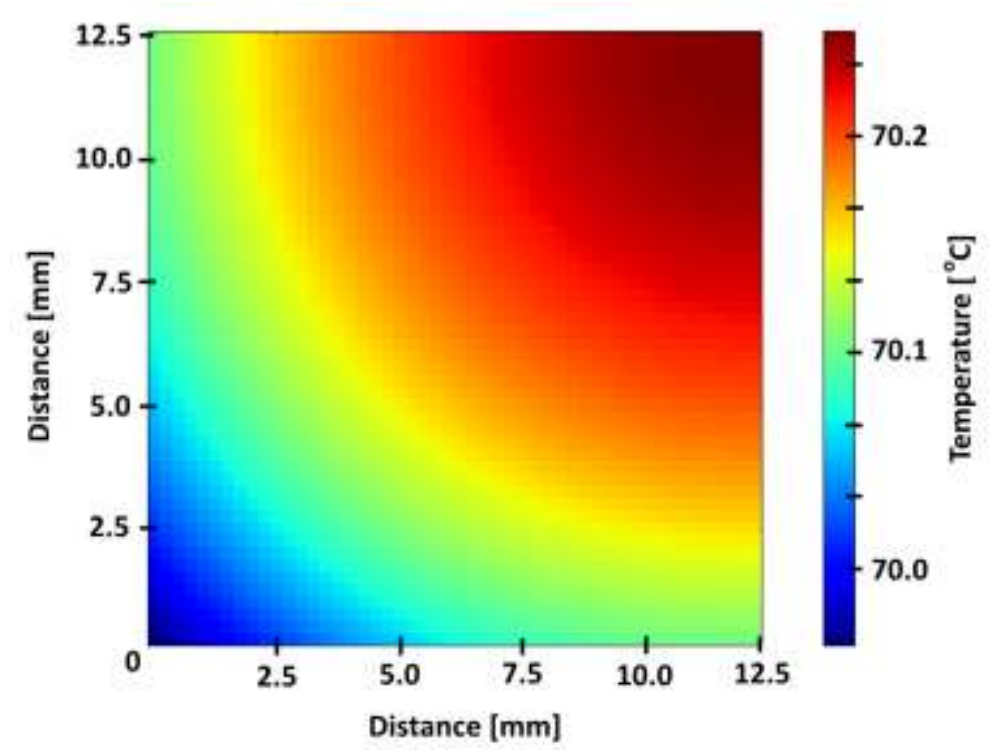

Figure 4: Lower left hand corner of two-dimensional system model

With the current system parameters and physical properties for myristic acid the model geometry very rapidly approaches that of a sphere, with only minor deviations at the outermost corners. For this reason the spherical representation was chosen for both finite difference models and the simplified model. This improves performance since the spherical model is effectively one dimensional. 


\section{Experimental}

The PCM measurement system is built using locally produced extruded polystyrene foam (ISOboard, South Africa). This material was chosen due to its particularly low thermal conductivity $\sim 0.024 \mathrm{~W} . \mathrm{m}^{-1} . \mathrm{K}^{-1}$, which allows extremely slow cooling of the sample. Structurally it is simply a cube of foam $(13 \mathrm{~cm} \times 13 \mathrm{~cm} \mathrm{x} 13$ $\mathrm{cm})$ with a small square cavity at its centre $(3 \mathrm{~cm} \times 3 \mathrm{~cm} \times 3 \mathrm{~cm})$. Molten PCM is cast into this cavity and the device is sealed. Embedded in the cavity are three very small thermocouples ( $\sim 0.8 \mathrm{~mm}$ diameter) and a small resistive heater. Fine gauge, fast response, K-type thermocouples supplied by OMEGA Engineering, Inc (U.S.A.) were used. These have a tolerance of $0.4 \%$ of the operating range $\left(<0.4{ }^{\circ} \mathrm{C}\right.$ in this case $)$ and a fast response time of 0.2 seconds. The device is shown schematically in Figure 5.

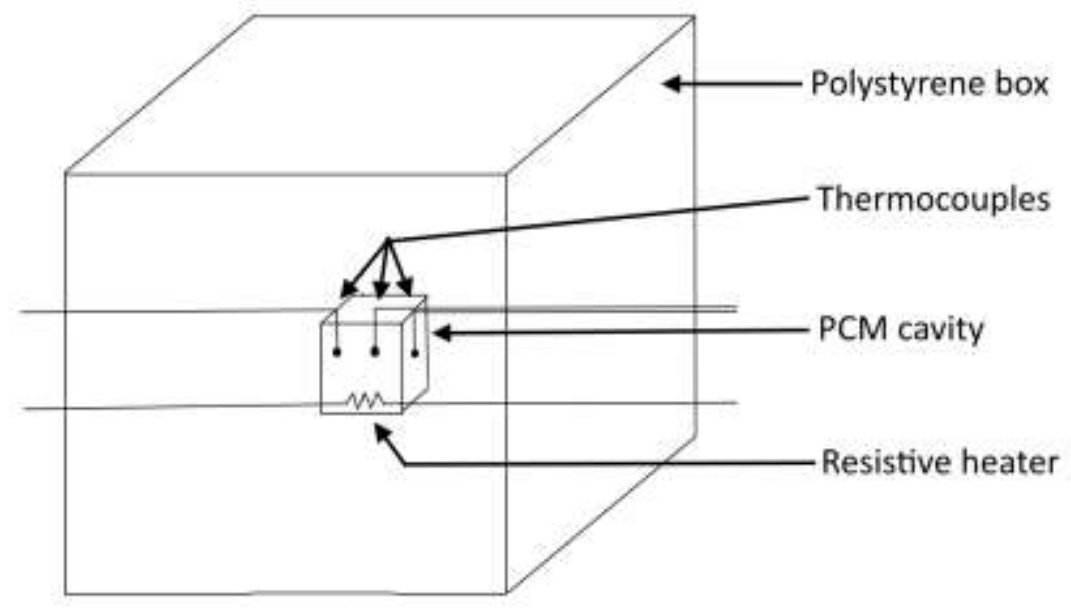

Figure 5: A schematic illustration of PCM measurement system

One thermocouple is positioned as close as possible to the centre of the cavity to measure the core temperature. The two remaining thermocouples are located on opposite sides of the inner cavity at the outer edge, i.e. at the PCM-foam interface. The polystyrene box is suspended in the air to allow equal cooling from all sides. Initially the cavity is resistively heated using a regulated power supply to a temperature beyond the melting point of the chosen PCM. Once a suitable temperature is achieved at the core, the heating is switched off and all the thermocouple measurements logged electronically as the system cools down. Since the molten PCM is in direct contact with the foam, there is negligible interfacial or contact resistance. The molten PCM does not penetrate into the closed cell foam. The 
myristic acid used in this experiment was obtained from Sigma-Aldrich (>95\% purity).

The heat capacities and enthalpy of fusion were measured using a Perkin-Elmer Differential Scanning Calorimeter (DSC 4000), whilst the thermal conductivity was determined with a Linseis Transient Hot Bridge instrument (THB-100).

\section{Results and discussion}

The thermocouple measurements for a single experiment are shown below in Figure 6.

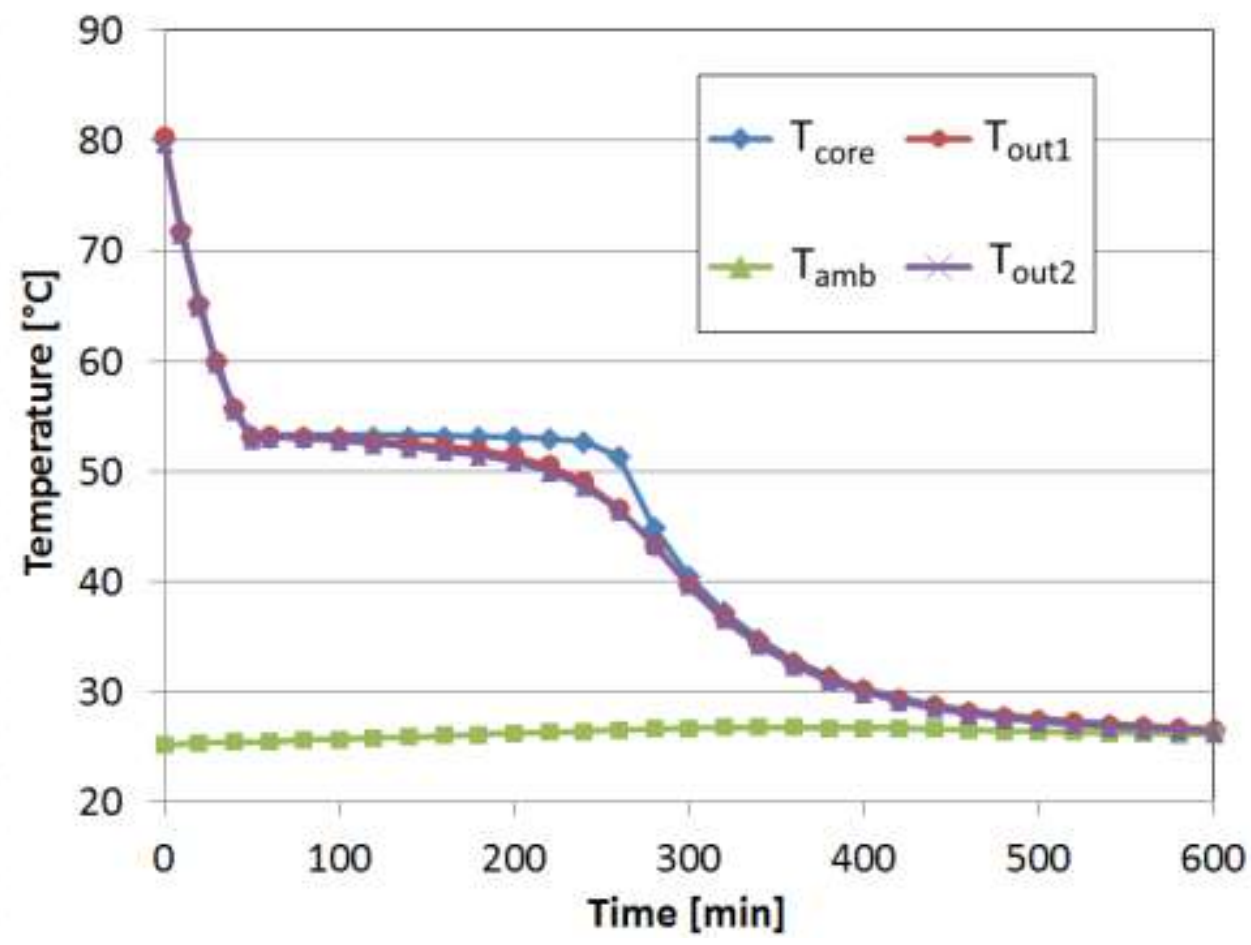

Figure 6: Experimentally measured temperatures

Ambient temperature is included to confirm that no significant drift was present, although this could easily be incorporated into the model. Since both outer temperatures exhibit identical behaviour only the core and one outer temperature were used for analysis. Despite the low thermal conductivity of the sample, the core and outer temperatures are remarkably similar during the liquid and solid regions of the curve. The only significant deviation is towards the final stages of solidification. This is not unexpected given the very slow cooling rate. The relevant fixed model parameters are specified in Table 1. 
Table 1: Model parameters

\begin{tabular}{|c|c|c|}
\hline Description & Value & Units \\
\hline Polystyrene thermal conductivity & 0.024 & $\mathrm{~W} \cdot \mathrm{m}^{-1} \cdot \mathrm{K}^{-1}$ \\
\hline PCM solid density & 990 & $\mathrm{~kg} \cdot \mathrm{m}^{-3}$ \\
\hline PCM liquid density & 861 & $\mathrm{~kg} \cdot \mathrm{m}^{-3}$ \\
\hline PCM liquid thermal conductivity & 0.6 & $\mathrm{~W} \cdot \mathrm{m}^{-1} \cdot \mathrm{K}^{-1}$ \\
\hline PCM melting point & 53.2 & ${ }^{\circ} \mathrm{C}$ \\
\hline Ambient temperature & 26.2 & $\mathrm{~mm}$ \\
\hline Inner cavity length & 30 & $\mathrm{~mm}$ \\
\hline Outer box length & 130 & $\mathrm{~mm}$ \\
\hline Length step size & 0.7 & $\mathrm{Min}$ \\
\hline Time step size & 2 & $\mathrm{~mm}$ \\
\hline
\end{tabular}

The polystyrene thermal conductivity was obtained from the supplier whilst the other properties were taken from literature [19]. The only exception is the liquid thermal conductivity, which is very difficult to measure accurately since it is highly dependent on convective effects. The given value was estimated from an analytical analysis of the temperature difference in the liquid region only. The prediction of the effective heat capacity model and residual error for the outer temperature are illustrated in Figure 7.

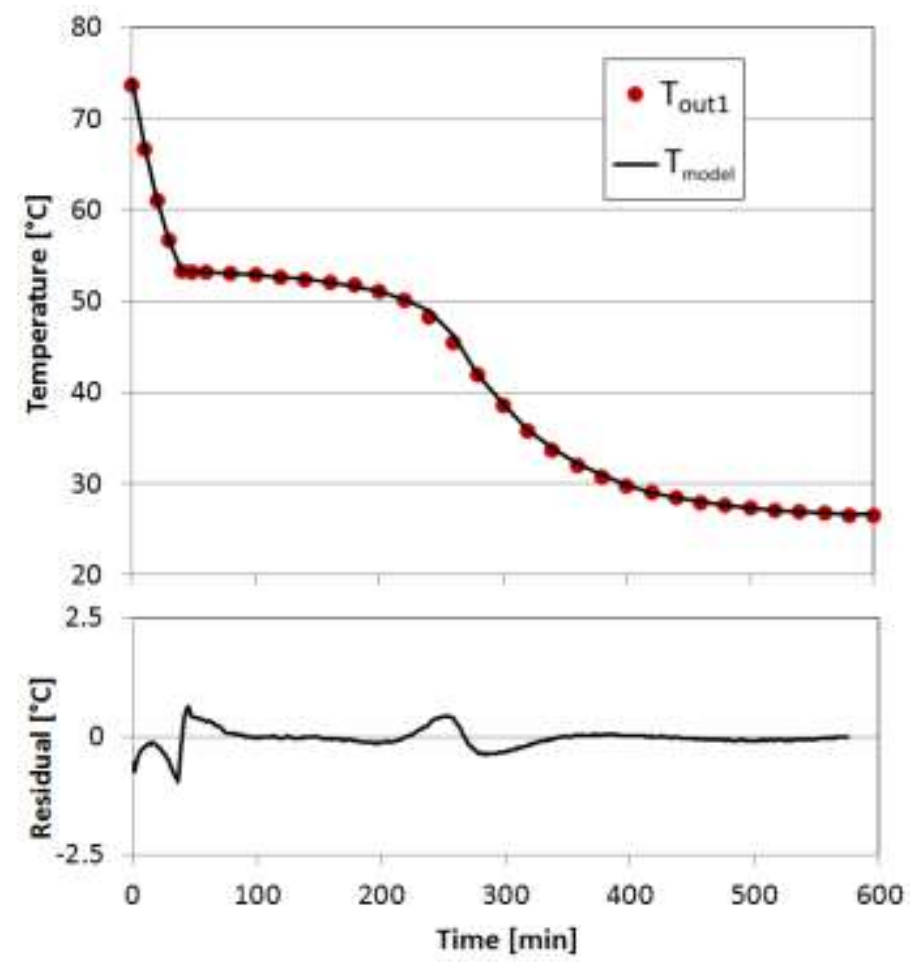

Figure 7: Fit result for the effective heat capacity model 
In general the fit is excellent for all three regions, i.e. liquid, phase change and solid. Not surprisingly the only regions where appreciable errors develop are at the inception and conclusion of phase change, i.e. at the points of transition from single to multi-phase. The results for the enthalpy method are demonstrated in Figure 8.

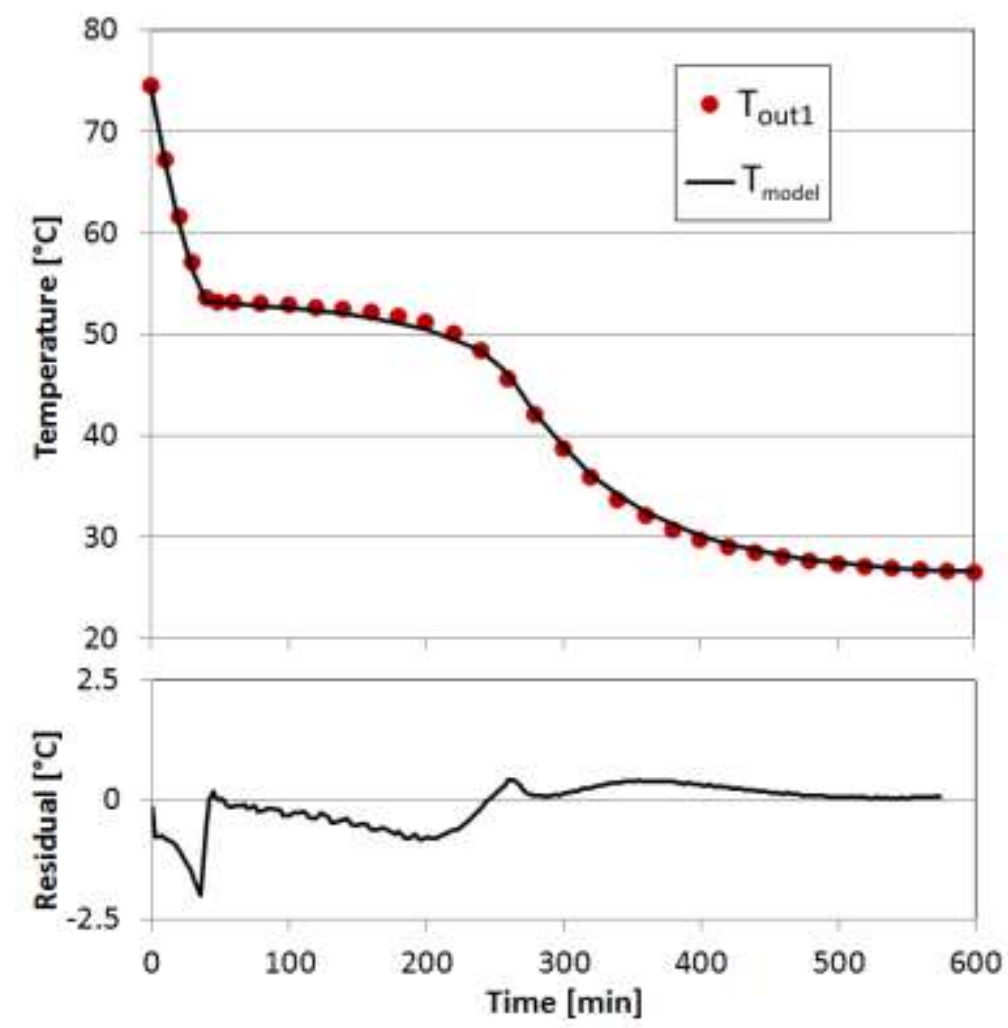

Figure 8: Fit result for the enthalpy model

The fit is also very good for all three regions but a significant difference is immediately evident from the residual error. During phase change the nodes are effectively held constant until each space interval is "filled" with solid material, i.e. until the melt fraction $f(T)$ proceeds from 0 to 1 , leading to a step change in model evolution. This stepping behaviour is more evident if this region is examined more closely as in Figure 9. 


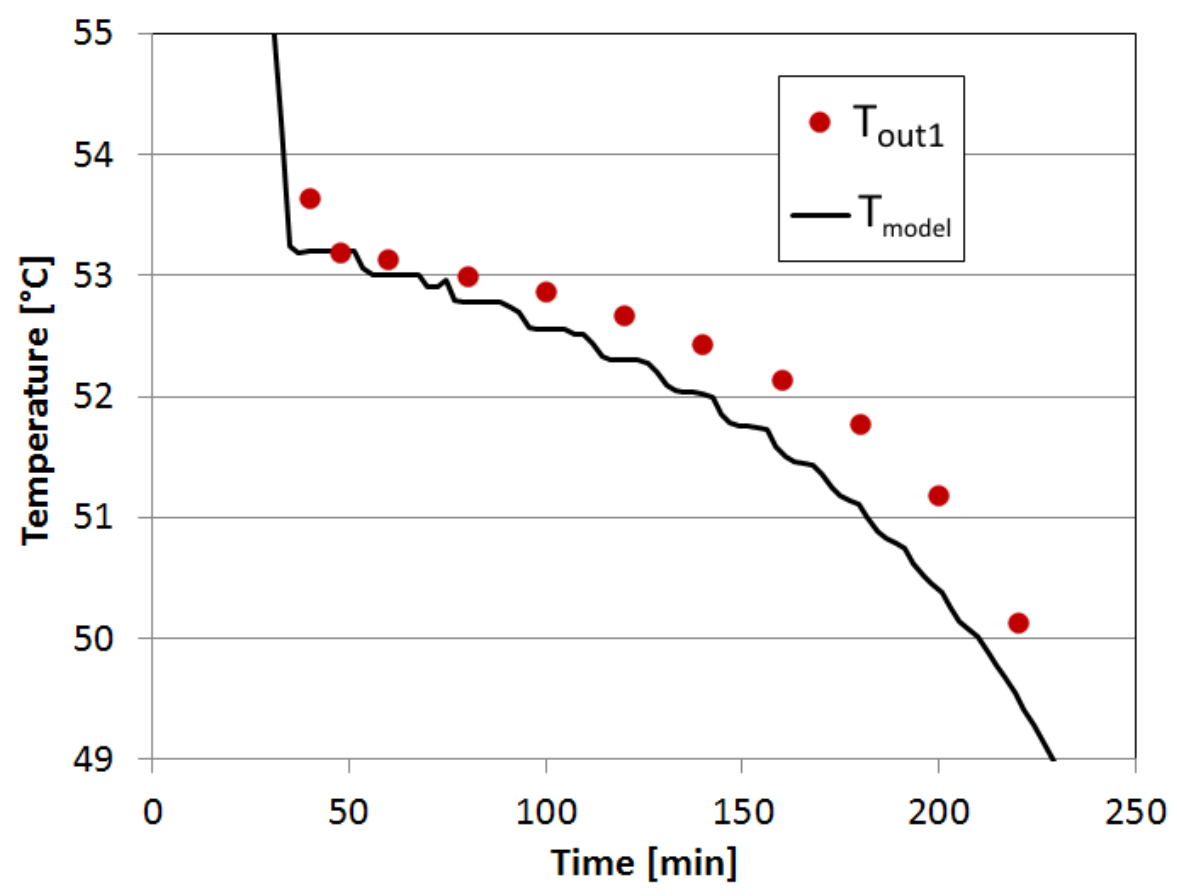

Figure 9: Stepping behaviour of the enthalpy model

Naturally this behaviour can be diminished by selecting a higher value for the number of length intervals, thus decreasing the size of these steps. However this would increase the time required for each simulation. Thus the choice of step size becomes a trade-off between simulation time and accuracy. The dependence of run times on both step sizes are demonstrated in Figure 10 below.

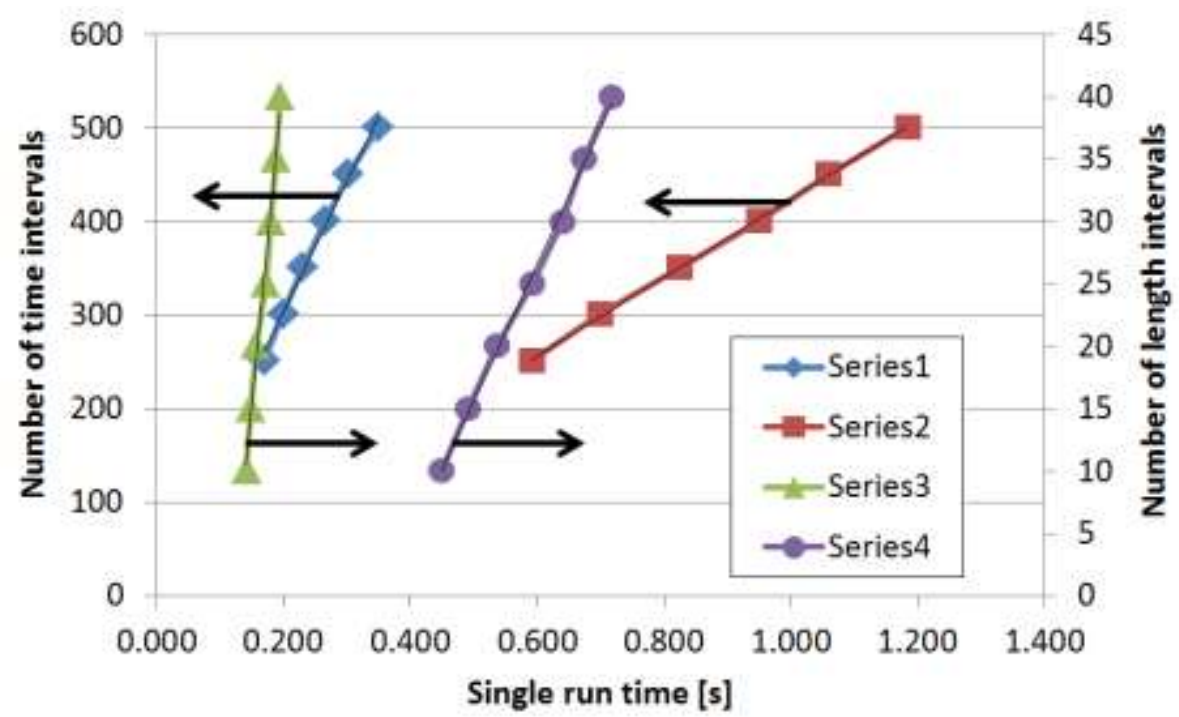

Figure 10: Run length 
As can be seen from this figure there is an almost linear decrease in the time required for a single run as a function of both parameters for both simulations. Thus for optimal performance the smallest number of intervals would be ideal. However as mentioned this creates a trade-off with model accuracy and the extent to which the model remains representative of the experimental measurement. In Figure 11 this trade-off becomes evident as the sum of the absolute error is plotted.

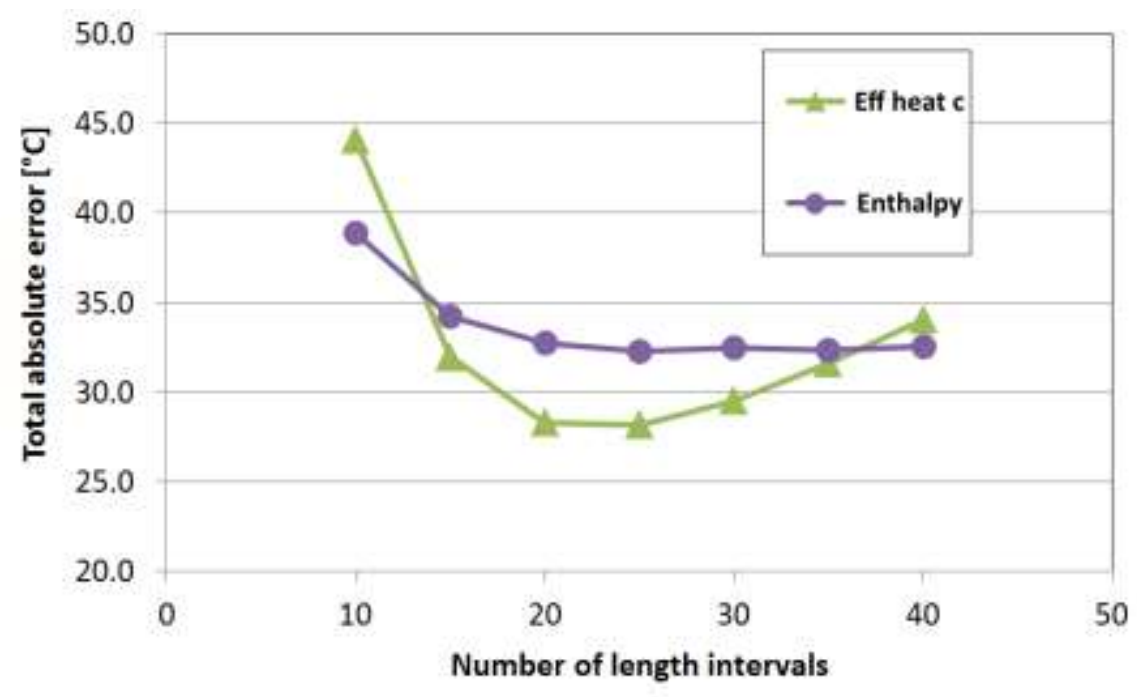

Figure 11: Total absolute error

In both cases the error begins to increase below around 25 intervals $(0.7 \mathrm{~mm}$ length step size), which is why this value was chosen. An additional factor which is seldom considered is the stability of the model in the two phase region. Whilst the use of the implicit difference scheme guarantees stability when only a single phase is present, the same is not true for the phase transition zone. For these methods a problem occurs due to the relative size of the step width compared to the melt/freeze zone. As the time steps are increased or the length steps reduced a point is reached where the model may calculate that freezing occurs across the entire sample length in a single step. In the original presentation Voller [27] stated that in most cases at most two iterations are required to achieve convergence. If the simulation is restricted to only two iterations it will become unstable as demonstrated in Figure 12, for the enthalpy method. 


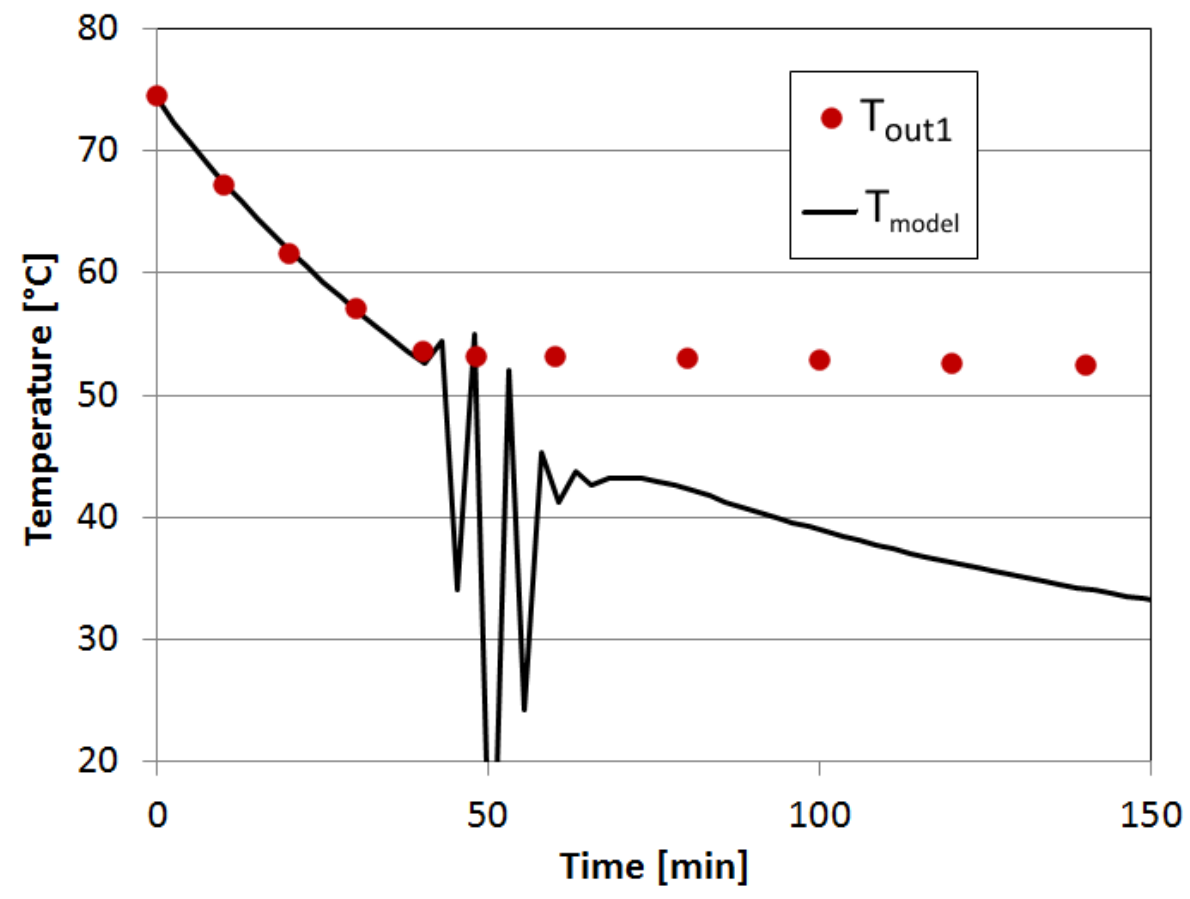

Figure 12: Instability of the enthalpy method

The case in Figure 12 occurs at a time step size of 1.85 min (232 time intervals) with $0.7 \mathrm{~mm}$ length steps. By trial and error it was found that increasing the number of iterations to seven guaranteed convergence up to very large time steps. However, the extra iterations increase the computational load which leads to longer run times. With only 101 time intervals and seven iterations, the average time per run is $6.7 \mathrm{~s}$. When two iterations are used just above the point of instability the time per run is 5.9 s. For this reason a step size of 2 min (251 time intervals) was chosen as a safe margin from instability without significantly increasing computational time by limiting the iterations to only two.

As mentioned earlier, in the enthalpy approach a slightly different scheme to the original was used to calculate the transition from liquid to solid properties (scaling by the melt fraction). This was found to make a negligible difference in the prediction error and performance. However, it was discovered (for both schemes) that if the difference between the solid and liquid phase properties are too large, in this case one order of magnitude, the enthalpy approach again becomes oscillatory or unstable and additional iterations must be added to ensure convergence. The heat capacity approach on the other hand shows no such shortcoming since it lacks the need for an 
iterative solution. It is also interesting to note the differences in the core temperature prediction for each model as illustrated in Figure 13.

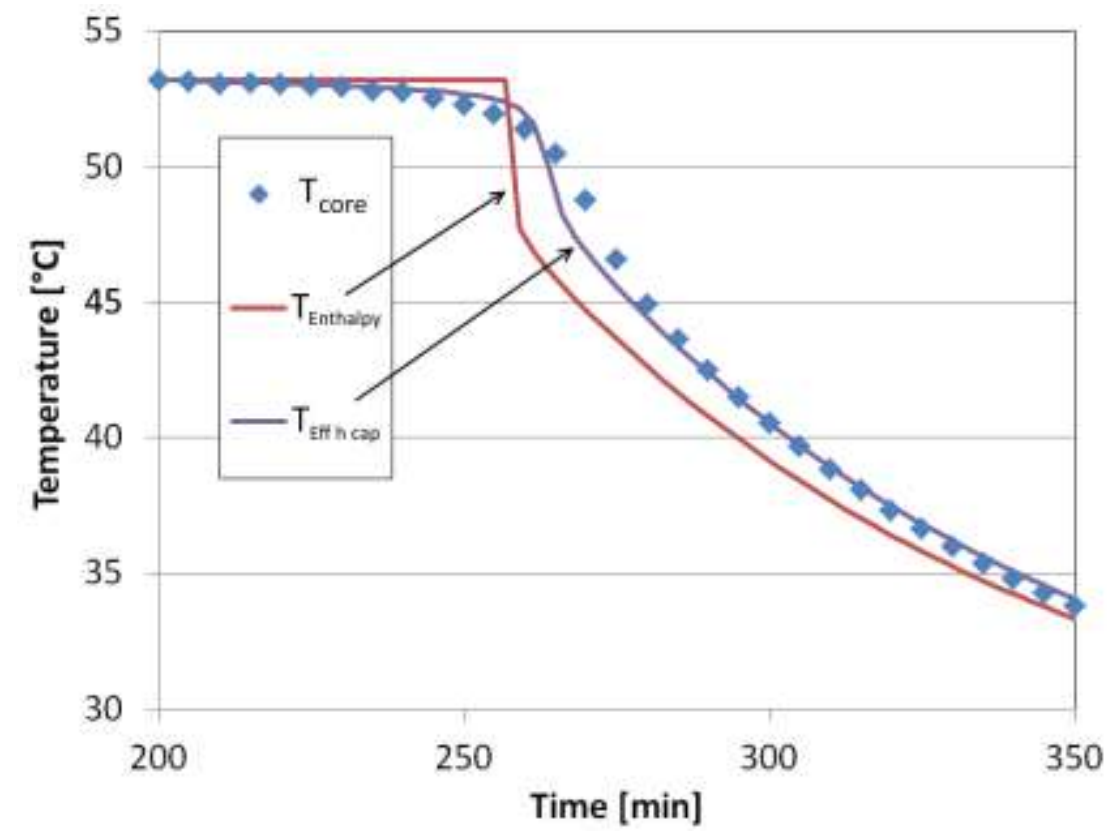

Figure 13: Core temperature predictions

As expected the enthalpy method predicts a very rapid, almost vertical transition for the core temperature at the point of solidification. When heat flow generation due to freezing ceases, the central temperature must transition very rapidly from its off-set condition to the temperature required for a single phase under conduction. However, since the effective heat capacity method undergoes a smooth transition due to the use of the normal distribution, its temperature changes more gradually. As mentioned earlier, from a kinetic perspective this is probably a more realistic representation. Thus one may have been inclined to assume that the measured core temperature is either not positioned perfectly or is acting as a nucleation site since it doesn't reflect the behaviour of the enthalpy method. This insight on the other hand suggests that the observed behaviour may, at least in part, be caused by a real effect. Another system insight is gained by considering the derivative of the outer temperature, calculated in Figure 14. 


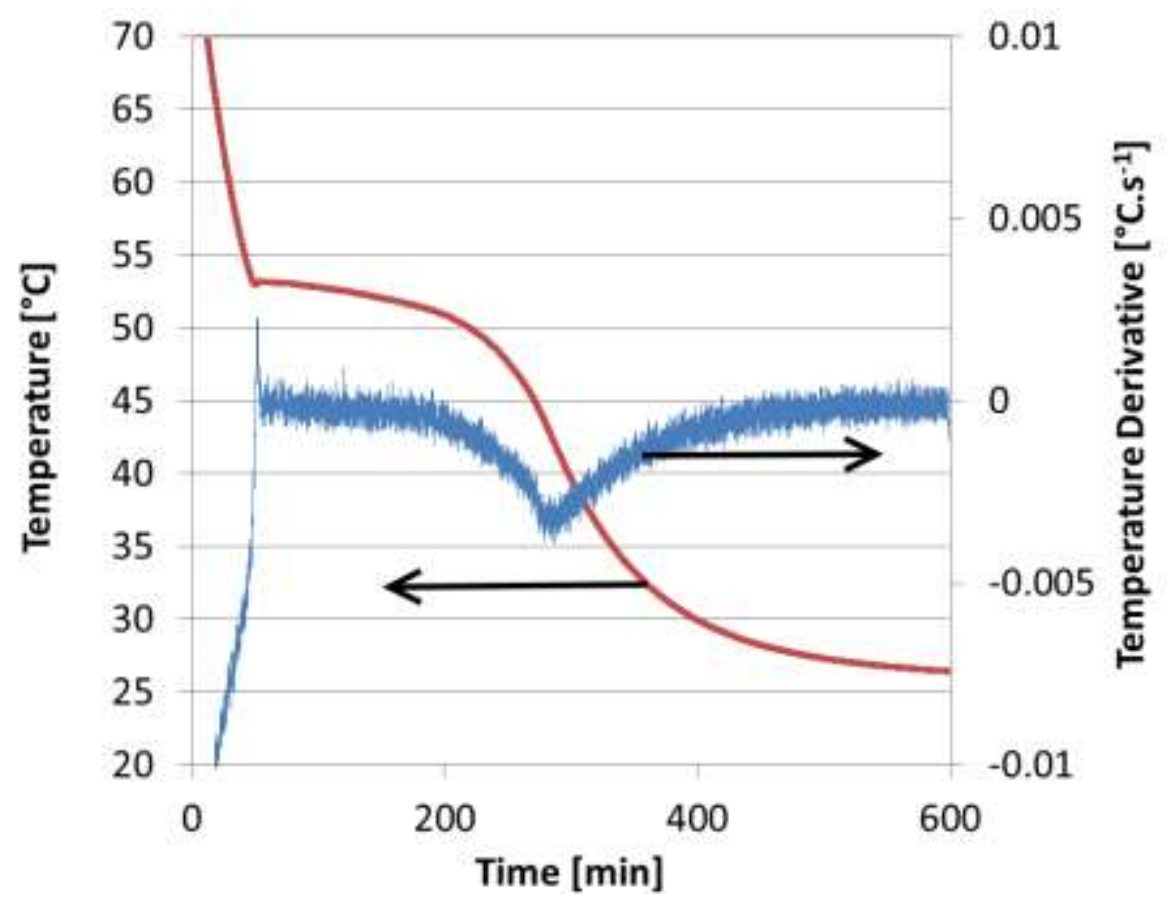

Figure 14: Derivative of outer temperature

From the point where the phase transition starts the derivative of the temperature remains essentially constant over a substantial time period ( $120 \mathrm{~min})$. Thus the heat flow rate from the surface during this period is approximately invariable; since the ambient temperature is constant the same can be said of the heat loss through the polystyrene box. Hence it was concluded that during this period the system is at steady state and the heat removed must be equal to the latent heat generated by freezing. This prompted the proposal of the third and much simpler model. It contains several assumptions in order to facilitate rapid solution.

As can be seen from Figure 6, the temperature difference between the outer and core temperatures during the single phase zones (both liquid and solid) are virtually zero. This is partially due to the very low cooling rate and allows these two regions to be very easily and adequately represented by the standard lumped parameter model. Hence the simplified approach merely aims to estimate the phase change enthalpy and the thermal conductivity of the solid and is only valid during the initial stages of solidification.

Initially the liquid phase is assumed to be homogenous and at the melting temperature while the heat flow is at steady state. This implies a linear temperature profile within the system, which is in effect comprised of two thermal resistances in 
series. The first is the growing solid and the second is the polystyrene box. All the heat leaving the system is presumed to be a result of the phase change. This is a fair assumption during the early stages of solidification when the interfacial temperature is still approximately equal to the melting temperature, i.e. very little sensible heat loss has occurred. Only once the solid fraction within the cavity has grown to a substantial value does this resistance become large enough to cause a significant deviation. The full derivation is given in Appendix A, which results in the following fully analytical expression:

$$
t=\frac{\rho_{s} \Delta H}{k_{s}\left(T_{\infty}-T_{m}\right)}\left[\frac{r^{3}}{3}\left(\frac{4 \pi k_{s}}{24 k_{p}}-\frac{1}{r_{0}}\right)+\frac{r^{2}}{2}-\frac{r_{0}^{3}}{3}\left(\frac{4 \pi k_{s}}{24 k_{p}}-\frac{1}{r_{0}}\right)-\frac{r_{0}^{2}}{2}\right]
$$

Where $r$ is the radius of the remaining liquid sphere and $t$ is the elapsed time. The roots of this equation can be solved at any time since the start of solidification to estimate the solid thickness. This in turn can be used to estimate the interfacial temperature. Thus the solution constitutes a progressive integral which at any given point in time is independent of the behaviour of the interfacial temperature in the future. This makes solving very rapid and in theory, only a single data point is required for the fit. However, in this investigation the interfacial temperature is estimated as a function of time and then the model parameters are adjusted to obtain the best fit over the chosen time period. This provides a much more robust estimate, which is shown in Figure 15.

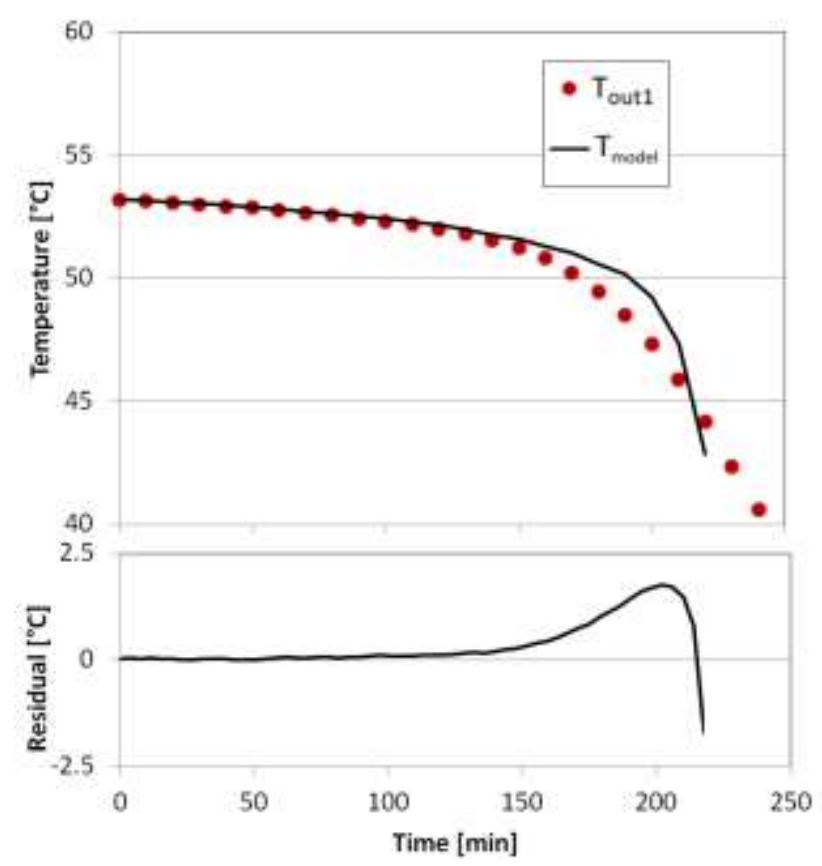

Figure 15: Fit result for the simplified analytical model 
As expected the fit is reasonable for the first 120 minutes of freezing but starts to deviate significantly beyond this point. Despite that fact that this fit may appear somewhat lacklustre it should be noted that the scale has been significantly reduced. It is a factor of three less than the one used in Figure 7 and 8 for the other methods. A cut-off point for the simulation was chosen as the time when the radius reaches $20 \%$ of its original value. Because of the spherical nature of the system (volume decreases with $r^{3}$ ) this constitutes virtually the entire melting time range. Furthermore it was found that the model could still provide an accurate property estimate using only the first 45 minutes of experimental data. This means that the experimental time can be drastically reduced and when combined with the two lumped parameter estimates (L.P.) of the heat capacities provides all four thermal properties.

A comparison of the overall performance of all three models is given in Table 2, along with the instrumental measurements and literature values for comparison.

Table 2: Thermal property estimates

\begin{tabular}{|c|c|c|c|c|c|}
\hline & $\begin{array}{l}\text { Eff. heat } \\
\text { capacity }\end{array}$ & Enthalpy & $\begin{array}{c}\text { Simple } \\
\text { analytical }\end{array}$ & $\begin{array}{l}\text { Instrument } \\
\text { DSC/THW }\end{array}$ & Literature \\
\hline Convergence time [s] & 40.0 & 126.5 & 10.3 & & \\
\hline Number of runs & 230 & 216 & 48 & & \\
\hline $\begin{array}{l}\text { Average absolute error } \\
\qquad\left[{ }^{\circ} \mathrm{C}\right]\end{array}$ & 0.137 & 0.333 & 0.195 & & \\
\hline $\begin{array}{c}\text { Enthalpy of fusion }\left[\mathrm{kJ} . \mathrm{kg}^{-}\right. \\
\left.{ }^{1}\right]\end{array}$ & 204.7 & 206.6 & 208.6 & 204.8 & $204.5[28]$ \\
\hline $\begin{array}{l}\text { Solid thermal cond. [W.m } \\
\left.{ }^{1} \cdot \mathrm{K}^{-1}\right]\end{array}$ & 0.39 & 0.31 & 0.36 & 0.22 & $\begin{array}{l}0.39 \\
{[33]}\end{array}$ \\
\hline $\begin{array}{l}\text { Solid heat capacity }\left[\mathrm{kJ}^{\mathrm{kg}}{ }^{-}\right. \\
\left.{ }^{1} \cdot \mathrm{K}^{-1}\right]\end{array}$ & 2.59 & 2.81 & $\begin{array}{l}2.96 \\
\text { (L.P.) }\end{array}$ & 2.72 & $\begin{array}{c}2.8 \\
{[28]}\end{array}$ \\
\hline $\begin{array}{c}\text { Liquid heat capacity } \\
{\left[\mathrm{kJ} \cdot \mathrm{kg}^{-1} \cdot \mathrm{K}^{-1}\right]}\end{array}$ & 2.45 & 2.40 & $\begin{array}{l}2.20 \\
\text { (L.P.) }\end{array}$ & 2.37 & $\begin{array}{c}2.4 \\
{[28]}\end{array}$ \\
\hline
\end{tabular}

It should be noted that the fit for the simple analytical model was only done across the phase change zone, which is approximately a third of the total data range used for the other two models. The values taken from literature were used as starting values in all three cases. The parameter estimation was done using the L-BFGS algorithm as 
implemented in SciPy [35, 36]. The whole data-processing chain was implemented in Python using the SciPy routines. Specifically the linear matrix equations were solved using the LAPACK routine "_gesv", which does LU decomposition with partial pivoting and row interchanges.

All models give similar results for the enthalpy of fusion which agrees well with the literature and measured values. Since this is a pure material, not a composite, the DSC result is expected to be very accurate and the same holds for the heat capacities. These are equally well predicted by all three approaches with the effective heat capacity method under-estimating the solid heat capacity slightly. However, for the solid thermal conductivity the THW technique underestimates the value fairly significantly due to the porous nature of the material despite being cast in liquid PCM. The crystal structure formed when freezing liquid myristic acid invariably contains voids since the density of the solid is higher than the liquid. All models predict this value well with only the enthalpy method under-predicting it slightly.

All models show a very low average absolute error, indicating that in all cases the predictions are within a third of a degree on average for the whole data set. The enthalpy method requires fewer runs for convergence but still shows a significantly increased convergence time. The reason for this is the requirement for an iterative solution, despite being restricted to only two. Since the time is more than double that of the effective heat capacity method other factors may also contribute to this, for example the solution speed of the linear matrix equations. The effective heat capacity method performs admirably considering that all four properties are delivered in a single $40 \mathrm{~s}$ fitting procedure with the lowest average error of all three.

As one would expect the simple analytic model outperforms both by a significant margin. This can be further reduced by restricting to fitting to the initial period (45 min), but the gain is not significant due to the already very basic nature of the calculation. The prediction accuracy of this simple model is comparable to the effective heat capacity method with an average error of less than one fifth of a degree.

\section{Conclusions and recommendations}

The measurement of thermal properties continues to be a challenge facing the development of PCM storage systems. Porous and particulate composites are being explored which increase the thermal conductivity but pose practical challenges for 
traditional analytical instruments. To overcome this issue a simple system has been developed to quickly and easily measure the thermal properties of these composite materials. In order to validate the approach a fundamental model is required. However modelling the transition from liquid to solid phases presents difficulties of its own.

Currently two main approaches are followed to represent the phase transition, namely the effective heat capacity method and the enthalpy method. The first uses a representation of the heat capacity function (i.e. enthalpy derivative curve) whilst the second employs a melt fraction which must be calculated iteratively. Very few studies have focused on comparing the performance of these two schemes and less, if any, on their relative ability to fit real experimental data. Furthermore the stability of these methods in the two phase region is rarely mentioned.

A performance comparison was done for these two approaches, as well as a third, simplified analytical model. The main criteria were convergence time, accuracy and stability. Despite the fact that an implicit difference scheme was used the enthalpy model is still susceptible to unstable or oscillatory behaviour in the two phase region. This may be triggered through the choice of step size or a large disparity between the liquid and solid properties. The effective heat capacity method did not show any such behaviour. In addition, due to the choice of a smooth function for the transition from liquid to solid, this method delivers more realistic behaviour as demonstrated for the core temperature.

The underlying reason for the instability of the enthalpy method is the requirement for an iterative solution. The number of iterations required are reduced by the use of the so-called "new source scheme" whereby the matrix coefficients at nodes where phase change is occurring are made arbitrarily large. But in effect the system still requires the tracking of the melt front through the use of liquid fraction to set these matrix coefficients and change the thermal properties from liquid to solid. This makes the approach slightly more difficult to implement than the effective heat capacity method which simply uses one continuous function.

Another significant drawback of the iterative solution is the increased computational load. The effective heat capacity method outperforms the enthalpy method by a factor of three, in terms of time required to achieve convergence. This takes into account not only the time required for a single run but the manner in which convergence take place since the models were fit to actual experimental data. All 
three models perform quite well in terms of accuracy. All three have an average prediction error of less than one third of a degree and estimate all four thermal properties to within an acceptable level of accuracy.

The best performing approach from a convergence time perspective is the analytical approach due to very basic calculation requirements. It can be used in conjunction with the lumped parameter method to rapidly and comparatively accurately predict all four thermal properties. In addition, it can reduce the time required for a single experimental significantly since only the initial portion of the phase change data is needed. Thus it is a viable option for the rapid determination of the thermal properties of phase change materials. However, for the current setup this is impractical and a more robust and comprehensive fit is sought which can provide additional insights such as the complete temperature distribution. This will enable a more comprehensive understanding of the material behaviour and properties. For this the effective heat capacity method is preferred above the enthalpy method.

Future work will focus on the thorough validation of the approach for parameter estimation, using a series of model compounds and composites.

\section{Acknowledgements}

This work is based on research supported by the South African Research Chairs Initiative (SARChI) of the Department of Science and Technology (DST) and the National Research Foundation (NRF). Any opinion, findings and conclusions or recommendations expressed in this material are those of the authors and therefore the NRF and the DST do not accept any liability with regard thereto.

\section{References}

[1] European Commission. Concentrating solar power - From research to implementation. Published in Luxembourg by Office for Official Publications of the European Communities; 2007. ISBN 978-92-79-05355-9. ec.europa.eu.

[2] Sargent \& Lundy LLC Consulting Group. Assessment of parabolic trough and power tower solar technology cost and performance forecasts. Report NREL/SR550-34440, NREL (National Renewable Energy Laboratory), Golden, CO,USA; 2003 
[3] Khare S, Dell'Amico M, Knight C, McGarry S. Selection of materials for high temperature latent heat energy storage. Sol. Energy Mater. Sol. Cells 2012;107:207.

[4] Kuravi S, Trahan J, Goswami DY, Rahman MM, Stefanakos EK. Thermal energy storage technologies and systems for concentrating solar power plants, Prog. Energy Combust. Sci. 2013;39:285-319.

[5] Zeng J, Zheng S, Yu S, Zhu F, Gan J, Zhu L, et al. Preparation and thermal properties of palmitic acid/polyaniline/exfoliated graphite nanoplatelets formstable phase change materials. Appl. Energy 2014;115:603-9.

[6] Zhang L, Zhu J, Zhou W, Wang J, Wang, Y. Thermal and electrical conductivity enhancement of graphite nanoplatelets on form-stable polyethylene glycol / polymethyl methacrylate composite phase change materials. Energy 2012;39:294302

[7] Fang Z, Fan L, Ding Q, Wang X, Yao X, Hou J, et al. Increased Thermal Conductivity of Eicosane-Based Composite Phase Change Materials in the Presence of Graphene Nanoplatelets. Energy Fuels 2013;27:4041-7.

[8] Ji H, Sellan DP, Pettes MT, Kong X, Ji J, Shi L, et al. Enhanced thermal conductivity of phase change materials with ultrathin-graphite foams for thermal energy storage. Energy Environ. Sci. 2014;7:1185-92.

[9] Li M, Wu Z, Tan J. Properties of form-stable paraffin/silicon dioxide/expanded graphite phase change composites prepared by sol-gel method. Appl. Energy 2012;92:456-61.

[10] Xiao X, Zhang $\mathrm{P}$, Li M. Thermal characterization of nitrates and nitrates/expanded graphite mixture phase change materials for solar energy storage. Energy Convers. Manage. 2013;73:86-94.

[11] Fan LW, Fang X, Wang X, Zeng Y, Xiao YQ, Yu ZT, et al. Effects of various carbon nanofillers on the thermal conductivity and energy storage properties of paraffin-based nanocomposite phase change materials. Appl. Energy 2013;110:163-72.

[12] Fauzi H, Metselaar HSC, Mahlia TMI, Silakhori M, Nur H. Phase change material: Optimizing the thermal properties and thermal conductivity of myristic acid/palmitic acid eutectic mixture with acid-based surfactants. Appl. Therm. Eng. 2013;60:261-5. 
[13] López-Navarro A, Biosca-Taronger J, Corberán JM, Peñalosa C, Lázaro, A, Dolado P, et al. Performance characterization of a PCM storage tank. Appl. Energy 2014;119:151-62.

[14] Kuznik F, David D, Johannes K, Roux JJ. A review on phase change materials integrated in building walls. Ren. and Sus. Energy Rev. 2011;15:370-91.

[15] Asseal MJ, Dix M, Gialou K, Vozar L, Wakeham WA. Application of the Transient Hot-Wire Technique to the Measurement of the Thermal Conductivity of Solids, Int. J. Thermophys. 2002;23:615-33.

[16] Jensen C, Xing C, Folsom C, Ban H, Phillips J. Design and Validation of a HighTemperature Comparative Thermal-Conductivity Measurement System, Int. J. Thermophys. 2012;33:311-29.

[17] Wulf R, Barth G, Gross U, Intercomparison of Insulation Thermal Conductivities Measured by Various Methods, Int. J. Thermophys. 2007;28:1679-92.

[18] Yinping Z, Yi J, Yi J. A simple method, the T -history method, of determining the heat of fusion, specific heat and thermal conductivity of phase-change materials. Meas. Sci. Technol. 1999;10:201-5.

[19] Zalba B, Marín JM, Cabeza LF, Mehling H. Review on thermal energy storage with phase change: materials, heat transfer analysis and applications. Appl. Therm. Eng. 2003;23:251-83.

[20] Verma P, Varun, Singal SK. Review of mathematical modeling on latent heat thermal energy storage systems using phase-change material. Ren. and Sus. Energy Rev. 2008;12:999-1031.

[21] Jegadheeswaran S, Pohekar SD. Performance enhancement in latent heat thermal storage system: A review. Ren. and Sus. Energy Rev. 2009;13:2225-44.

[22] Zhu N, Ma Z, Wang S. Dynamic characteristics and energy performance of buildings using phase change materials: A review. Energy Convers. Manage. 2009;50:3169-81.

[23] Agyenim F, Hewitt N, Eames P, Smyth M. A review of materials, heat transfer and phase change problem formulation for latent heat thermal energy storage systems (LHTESS). Ren. and Sus. Energy Rev. 2010;14:615-28.

[24] Zhou D, Zhao CY, Tian Y. Review on thermal energy storage with phase change materials (PCMs) in building applications. Appl. Energy 2012;92:593-605.

[25] Lui S, Li Y, Zhang Y. Mathematical solutions and numerical models employed 
for the investigations of PCMs' phase transformations. Ren. and Sus. Energy Rev. 2014;33:659-74.

[26] Dutil Y, Rousse DR, Salah NB, Lassue S, Zalewski L. A review on phase-change materials: Mathematical modeling and simulations. Ren. and Sus. Energy Rev. 2011;15:112-30.

[27] Voller VR. Fast implicit finite-difference method for the analysis of phase change problems. Numer. Heat Transfer, Part B 1990;17:155-69.

[28] Abhat A. Low temperature latent heat thermal energy storage: heat storage materials. Sol. Energy 1983;30:313-32.

[29] Sari A, Kaygusuz K. Thermal performance of myristic acid as a phase change material for energy storage application. Renewable Energy 2001;24:303-17.

[30] Sari A. Eutectic mixtures of some fatty acids for low temperature solar heating applications: Thermal properties and thermal reliability. Appl. Therm. Eng. 2005;25:2100-7.

[31] Duo M, Lijiu W. Characterization and Thermal Conductivity of Modified Graphite/Fatty Acid Eutectic/PMMA Form-Stable Phase Change Material. J. Wuhan University of Technology-Mater. Sci. Ed. 2013;28:586-91.

[32] Sari A, Alkan C, Altintaş A. Preparation, characterization and latent heat thermal energy storage properties of micro-nanoencapsulated fatty acids by polystyrene shell. Appl. Therm. Eng. 2014;73:1160-8.

[33] Bayram Ü, Aksöz, Maraşh N. Temperature dependency of thermal conductivity of solid phases for fatty acids. J. Therm. Anal. Calorim. 2014;118:311-21.

[34] Sharma A, Tyagi VV, Chen CR, Buddhi D. Review on thermal energy storage with phase change materials and applications. Ren. and Sus. Energy Rev. 2009;13:318-45.

[35] Byrd RH, Lu P, Nocedal J, A Limited Memory Algorithm for Bound Constrained Optimization. SIAM Journal on Scientific and Statistical Computing 1995;16:1190-208.

[36] Zhu C, Byrd RH, Nocedal J, L-BFGS-B: Algorithm 778: L-BFGS-B, FORTRAN routines for large scale bound constrained optimization. ACM Transactions on Mathematical Software 1997;23: 550-60. 


\section{Appendix A}

A differential segment of a sphere is shown in Figure A1.

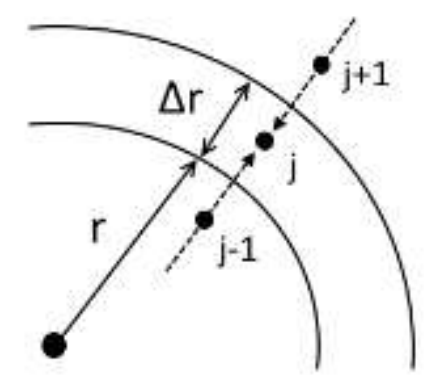

Figure A1: Finite difference of spherical segment

The complete energy balance for this segment around node $\mathrm{j}$ at time $\mathrm{p}+1$ is given by:

$$
\begin{gathered}
4 \pi r^{2} k\left(\frac{T_{j-1}^{p+1}-T_{j}^{p+1}}{\Delta r}\right)+4 \pi(r+\Delta r)^{2} k\left(\frac{T_{j+1}^{p+1}-T_{j}^{p+1}}{\Delta r}\right) \\
=\left(\frac{4}{3} \pi(r+\Delta r)^{3}-\frac{4}{3} \pi r^{3}\right) \rho \frac{h_{j}^{p+1}-h_{j}^{p}}{\Delta t}
\end{gathered}
$$

For the effective heat capacity (EFC) method this becomes:

$$
\begin{gathered}
4 \pi r^{2} k\left(\frac{T_{j-1}^{p+1}-T_{j}^{p+1}}{\Delta r}\right)+4 \pi(r+\Delta r)^{2} k\left(\frac{T_{j+1}^{p+1}-T_{j}^{p+1}}{\Delta r}\right) \\
=\left(\frac{4}{3} \pi(r+\Delta r)^{3}-\frac{4}{3} \pi r^{3}\right) \rho C p_{s} g(T) \frac{T_{j}^{p+1}-T_{j}^{p}}{\Delta t}
\end{gathered}
$$

For the enthalpy method the expression changes to:

$$
\begin{aligned}
& 4 \pi r^{2} k\left(\frac{T_{j-1}^{p+1}-T_{j}^{p+1}}{\Delta r}\right)+4 \pi(r+\Delta r)^{2} k\left(\frac{T_{j+1}^{p+1}-T_{j}^{p+1}}{\Delta r}\right) \\
= & \left(\frac{4}{3} \pi(r+\Delta r)^{3}-\frac{4}{3} \pi r^{3}\right)\left[\rho C p \frac{T_{j}^{p+1}-T_{j}^{p}}{\Delta t}+\rho \Delta H \frac{f_{j}^{p+1}-f_{j}^{p}}{\Delta t}\right]
\end{aligned}
$$

The expansion of the quadratic and cubic terms:

$$
\begin{gathered}
(r+\Delta r)^{2}=r^{2}+2 r \Delta r+\Delta r^{2} \\
(r+\Delta r)^{3}-r^{3}=3 r^{2} \Delta r+3 r \Delta r^{2}+\Delta r^{3}
\end{gathered}
$$

Neglecting the higher order differential terms $\left(\Delta \mathrm{r}^{3} \approx \Delta \mathrm{r}^{2} \approx 0\right)$, the EFC expression becomes: 


$$
r^{2}\left(\frac{T_{j-1}^{p+1}-T_{j}^{p+1}}{\Delta r}\right)+\left(r^{2}+2 r \Delta r\right)\left(\frac{T_{j+1}^{p+1}-T_{j}^{p+1}}{\Delta r}\right)=r^{2} \Delta r \frac{\rho C p_{s}}{k} g(T) \frac{T_{j}^{p+1}-T_{j}^{p}}{\Delta t}
$$

Or

$$
\frac{r^{2}}{\Delta r}\left(T_{j+1}^{p+1}+T_{j-1}^{p+1}-2 T_{j}^{p+1}\right)+2 r\left(T_{j+1}^{p+1}-T_{j}^{p+1}\right)=r^{2} \Delta r \frac{\rho C p_{s}}{k \Delta t} g(T)\left(T_{j}^{p+1}-T_{j}^{p}\right)
$$

Grouping similar and since $\alpha=\mathrm{k} / \rho \mathrm{Cp}$

$$
-\frac{\alpha \Delta t}{\Delta r^{2}} T_{j-1}^{p+1}+\left(g(T)+\frac{2 \alpha \Delta t}{\Delta r^{2}}+\frac{2 \alpha \Delta t}{r \Delta r}\right) T_{j}^{p+1}-\left(\frac{\alpha \Delta t}{\Delta r^{2}}+\frac{2 \alpha \Delta t}{r \Delta r}\right) T_{j+1}^{p+1}=g(T) T_{j}^{p}
$$

For the boundary cases, when $\mathrm{j}+1=\mathrm{j}-1$ :

$$
\left(g(T)+\frac{2 \alpha \Delta t}{\Delta r^{2}}+\frac{2 \alpha \Delta t}{r \Delta r}\right) T_{j}^{p+1}-\left(\frac{2 \alpha \Delta t}{\Delta r^{2}}+\frac{2 \alpha \Delta t}{r \Delta r}\right) T_{j+1}^{p+1}=g(T) T_{j}^{p}
$$

At the polystyrene interface the conduction leaving the system may be calculated as:

$$
q=k_{p}\left(\frac{L_{y}^{2}-p^{2}}{2 L_{x} \ln \left(L_{y} / p\right)}\right)\left(T_{\infty}-T_{i}\right)=k_{p} \beta\left(T_{\infty}-T_{i}\right)
$$

Where $L_{y}, L_{x}$, and $p$ are the dimensions of the polysterene box (outer height, half width and cavity height respectively). These are all constant and can be replaced by a single constant $\beta$, with $k_{p}$ being the thermal conductivity of the polystyrene. The energy balance at this outer node becomes:

$$
\begin{aligned}
k_{p} \beta\left(T_{\infty}-T_{j}^{p+1}\right) & +4 \pi r^{2} k\left(\frac{T_{j-1}^{p+1}-T_{j}^{p+1}}{\Delta r}\right) \\
& =\left(\frac{4}{3} \pi\left(r+\frac{\Delta r}{2}\right)^{3}-\frac{4}{3} \pi r^{3}\right) \rho C p_{s} g(T) \frac{T_{j}^{p+1}-T_{j}^{p}}{\Delta t}
\end{aligned}
$$

Simplifying and neglecting higher order differential terms:

$$
\frac{k_{p} \beta \Delta r}{4 \pi r^{2} k}\left(T_{\infty}-T_{j}^{p+1}\right)+\left(T_{j-1}^{p+1}-T_{j}^{p+1}\right)=\frac{\Delta r^{2}}{2 \alpha \Delta t} g(T)\left(T_{j}^{p+1}-T_{j}^{p}\right)
$$

Grouping similar:

$$
\begin{gathered}
{\left[g(T)+\frac{2 \alpha \Delta t}{\Delta r^{2}}\right.} \\
\left.+\left(\frac{k_{p} \beta}{4 \pi r^{2} k}\right)\left(\frac{2 \alpha \Delta t}{\Delta r}\right)\right] T_{j}^{p+1}-\frac{2 \alpha \Delta t}{\Delta r^{2}} T_{j-1}^{p+1} \\
=g(T) T_{j}^{p}+\left(\frac{k_{p} \beta}{4 \pi r^{2} k}\right)\left(\frac{2 \alpha \Delta t}{\Delta r}\right) T_{\infty}
\end{gathered}
$$

Similarly the expression for the enthalpy method is: 


$$
\begin{aligned}
& \frac{r^{2}}{\Delta r}\left(T_{j+1}^{p+1}+T_{j-1}^{p+1}-2 T_{j}^{p+1}\right)+2 r\left(T_{j+1}^{p+1}-T_{j}^{p+1}\right) \\
& =\left(\frac{r^{2} \Delta r}{k}\right)\left[\frac{\rho C p}{\Delta t}\left(T_{j}^{p+1}-T_{j}^{p}\right)+\frac{\rho \Delta H}{\Delta t}\left(f_{j}^{p+1}-f_{j}^{p}\right)\right]
\end{aligned}
$$

This can be simplified and grouped to give:

$$
\begin{aligned}
-\frac{\alpha \Delta t}{\Delta r^{2}} T_{j-1}^{p+1}+ & \left(1+\frac{2 \alpha \Delta t}{\Delta r^{2}}+\frac{2 \alpha \Delta t}{r \Delta r}\right) T_{j}^{p+1}-\left(\frac{\alpha \Delta t}{\Delta r^{2}}+\frac{2 \alpha \Delta t}{r \Delta r}\right) T_{j+1}^{p+1} \\
& =T_{j}^{p}-\frac{\Delta H}{C p}\left(f_{j}^{p+1}-f_{j}^{p}\right)
\end{aligned}
$$

With boundary cases:

$$
\left(1+\frac{2 \alpha \Delta t}{\Delta r^{2}}+\frac{2 \alpha \Delta t}{r \Delta r}\right) T_{j}^{p+1}-\left(\frac{2 \alpha \Delta t}{\Delta r^{2}}+\frac{2 \alpha \Delta t}{r \Delta r}\right) T_{j+1}^{p+1}=T_{j}^{p}-\frac{\Delta H}{C p}\left(f_{j}^{p+1}-f_{j}^{p}\right)
$$

And

$$
\begin{aligned}
\frac{k_{p} \beta \Delta r}{4 \pi r^{2} k}\left(T_{\infty}-\right. & \left.T_{j}^{p+1}\right)+\left(T_{j-1}^{p+1}-T_{j}^{p+1}\right) \\
= & \frac{\Delta r^{2}}{2 k}\left[\frac{\rho C p}{\Delta t}\left(T_{j}^{p+1}-T_{j}^{p}\right)+\frac{\rho \Delta H}{\Delta t}\left(f_{j}^{p+1}-f_{j}^{p}\right)\right]
\end{aligned}
$$

Which can be grouped as:

$$
\begin{aligned}
& {\left[1+\frac{2 \alpha \Delta t}{\Delta r^{2}}+\left(\frac{k_{p} \beta}{4 \pi r^{2} k}\right)\left(\frac{2 \alpha \Delta t}{\Delta r}\right)\right] T_{j}^{p+1}-\frac{2 \alpha \Delta t}{\Delta r^{2}} T_{j-1}^{p+1}} \\
& =T_{j}^{p}+\left(\frac{k_{p} \beta}{4 \pi r^{2} k}\right)\left(\frac{2 \alpha \Delta t}{\Delta r}\right) T_{\infty}-\frac{\Delta H}{C p}\left(f_{j}^{p+1}-f_{j}^{p}\right)
\end{aligned}
$$

Finally the simplified, analytical model can be found as follows, the total heat flow out of the system at steady state is given by:

$$
q=\frac{\Delta T}{\sum R}=\frac{T_{m}-T_{\infty}}{R_{1}+R_{2}}
$$

For the cavity, assumed spherical as with other models, the resistance to conduction through the solid phase is given by:

$$
R_{1}=\frac{1}{4 \pi k}\left(\frac{1}{r}-\frac{1}{r_{0}}\right)
$$

The same expression used previously for the polystyrene box is used:

$$
R_{2}=\frac{1}{k_{p} \beta}
$$

If it is assumed that all the heat lost is attributed to the phase change:

$$
q=\rho \Delta H \frac{d V}{d t}=-4 \pi r^{2} \rho \Delta H \frac{d r}{d t}
$$


Thus

$$
-4 \pi r^{2} \rho \Delta H \frac{d r}{d t}=\frac{T_{m}-T_{\infty}}{R_{1}+R_{2}}=\frac{T_{m}-T_{\infty}}{\frac{1}{4 \pi k}\left(\frac{1}{r}-\frac{1}{r_{0}}\right)+\frac{1}{k_{p} \beta}}
$$

The expression can be integrated to give:

$$
t=\frac{\rho_{s} \Delta H}{k_{s}\left(T_{\infty}-T_{m}\right)}\left[\frac{r^{3}}{3}\left(\frac{4 \pi k_{s}}{24 k_{p}}-\frac{1}{r_{0}}\right)+\frac{r^{2}}{2}-\frac{r_{0}^{3}}{3}\left(\frac{4 \pi k_{s}}{24 k_{p}}-\frac{1}{r_{0}}\right)-\frac{r_{0}^{2}}{2}\right]
$$

This may be used to determine the radius of the remaining liquid phase and the heat flow out at any given time, which in turn can be used in conjunction with $\mathrm{R}_{1}$ or $\mathrm{R}_{2}$ to calculate the interfacial temperature:

$$
T_{i}=T_{\infty}+\frac{q}{k_{p} \beta}
$$

